\title{
Palliative Care and Care for the Aged:An Achievable Reality in Bangladesh
}

\author{
N Ahmed ${ }^{1}$
}

World at present is experiencing a health transition with a rapidly rising burden of chronic and incurable diseases in one hand and on the other, a demographic transition in terms of increasing life expectancy. Infections and nutritional deficiencies are receding as leading contributors to death and disability in most of the countries. Cardiovascular diseases, cancers, diabetes, respiratory diseases, neuropsychiatry ailments, and other chronic diseases are becoming major burden of diseases and consequent mortality. In 2008, 63\% (36 of 57 million) deaths worldwide occurred due to Non-Communicable Diseases (NCDs) and nearly $80 \%$ of these deaths, i.e. 29 millions took place in low and middle income countries. NCD deaths are projected to increase by $15 \%$ globally between 2010 and 2020 causing for 44 million deaths! During this same period, the population of older person is growing at a rate of 2.6 per cent per year, considerably faster than the population as a whole which is increasing at 1.1 per cent annually.

Bangladesh is also in the same phase of demographic and disease transition. Chronic diseases accounted for 53\% of all deaths in 2005. The share of population above the age of 60 is growing at a rapid rate. In 2005, the total number of people aged 60 years and above was 5.64 million (about 5 $\%$ of the total population) and this number is expected to increase to 14.6 million (about $9 \%$ of the total population) by the year 2025 .

Looking at the present medical establishment, with its hospital-centered services, one can easily understand that it is geared basically to look after patients with acute illness. This acute-care orientation is reflected in the current emphasis on illness diagnosis, patient-initiated consultations, and curative and/or symptom relieving treatments. Many hospitals in state a policy of not admitting incurable patients, emphasizing that these are places of cure.

People dying of these "wrong conditions" and their careers, whether family, social or professionals are being increasingly frustrated by the major obstacles to accessing appropriate care.

Programmes for the prevention and control of these major non-communicable diseases chronic diseases are particularly difficult because it needs to adopt a 'life span' approach, attempting to reduce risks at different stages of life through appropriate multidimensional public health interventions. The ultimate intervention would be, well known in the modern medicine, as Palliative Care' and would have made a significant differences in the end- of life of these patients suffering from life limiting and incurable illnesses. This is particularly true for diseases like

1. Nezamudelin Ahmad, Professor \& Project Coordinator, Centre for Palliative Care, BSMMU. cancers which are predicted to become an increasingly important cause of morbidity and mortality in the next few decades, all over the world. Unfortunately, while the world has the knowledge and experience to control pain and diminish suffering significantly for these patients and their families, palliative care is not available for most of the global population. In fact, satisfactory palliative care does not exist in many countries where the needs are greatest and where it is probably the only realistic therapy or care to offer.

Palliative Care (derived from Latin palliare, to cloak) is a relatively new era of modern healthcare that focuses on relieving and preventing the suffering of patients with life limiting or incurable illnesses.

It is a concept of "active total care that improves the quality of life of patients and their families facing the problems associated with life-threatening illnesses, through the prevention and relief of suffering by means of early identification, impeccable assessment and treatment of pain and other problems, physical, psychosocial and spiritual". (World Health Organization). It is not solely about clinical skill and medical service to meet the psychosomatic need of the incurably ill patients whose life span is limited, but is also about the social and spiritual dimension of professional care. Palliative care should be seen as part of a continuum of care involving all the components of prevention and management of chronic diseases. Palliative care services, if implemented in a rational public health way, would quickly and dramatically improve the quality of life for cancer sufferes, other patients dying of chronic diseases, the elderly terminally ill and people living with AIDS. The success to implement this care in marginalized population, what is needed is the political will to act, educate and train the health professionals, involve the community and make the necessary affordable drugs easily available. The World Health Organization (WHO) has pioneered a public health strategy to integrate palliative care into existing healthcare systems. This offers the best approach for translating this new knowledge and skills into evidence-based, costeffective interventions that can reach everyone in the population, when incorporated by governments into all levels of their healthcare systems and owned by the community. It needs to be appreciated that most of the patients in need of palliative care and long term care will have to be looked after by health care professionals at the primary health care level. If the doctors and nurses at the field level are trained to improve their clinical and communication skills and are supported by a works of trained community workers and home care nurses, it can improve the care of the majority of these patients tremendously. The training of general practitioners and other doctors in rural areas in pain relief and 
palliative care are important as well as capacity building among the community volunteers and nurses. Emotional support, spiritual support, prophylaxis for avoiding bedsores, appropriate food, changing of bandages, etc are some of the important areas in which the family and local community can make a positive impact when empowered to do so. Establishment of adequate and accessible palliative care facilities in the community will also mean freeing expensive hospital beds from incurable patients. It will also culturally be the most important for reassuring the citizen's dignified death where they want it most, namely at home. Good quality community based home care services can help in reversing the present trend of materially and emotionally expensive institutionalized dying. Older people are often overlooked in the design of health care provision, and yet they often have the greatest and most complex needs. The needs of the elderly are becoming a concern to palliative care programs all over the world. This is because the same model of care can be adopted with some fundamental modification and integration. It must be appreciated and acknowledged that while modern medicine can often save a patient from a potentially fatal heart attack, few early cancers, and most bacterial disease, the survivors will eventually likely to go on to develop advanced end stage diseases such as heart failure, dementia, debilitating arthritis or Parkinson's disease at an old age. To provide proper care, clinicians must understand the unique needs of this age group as well as how the ageing process affects the delivery of health care to the elderly. Improvement in symptoms must be balanced with maintaining functional status and maximizing Quality of Life --- a challenge that makes care of the older adults sometimes difficult but always rewarding. Palliative care providers must learn to balance the multiple comorbid conditions of patients with chronic, eventually fatal illnesses. This re-framing of illnesses as the summed consequences of a multiple of chronic diseases is often difficult for clinicians as major medical training focuses on cure of discrete illnesses.

\section{References}

1. International Perspectives on Public Health and Palliative Care Edit by Libby salnow, Suresh Kumar and Allan Kellehear 2012 dge Studies in Public Health London and Newyork.

2. Rahman, Masud Ibn and Ali, Amin Masud, Population Aging and Its Implications in Bangladesh (February 25, 2009). Jahangirnagar Review Part II Social Science, Vol. XXXI, 2007. Available at SSRN: http://ssrn.com/abstract=1349447.

3. MR Rajgopal, David Mazza, Arthur G. Lipman Pain and Palliative Care in the developing World and Marginalized Populationa a global challenge in Jour nal of pain \& Pain
In elderly patients cure, is rarely an option. Instead, the goal is to maximize function and Quality of Life. Bangladesh is estimated to have more than 1 million patients with cancer at any point of time along with an equal number of other mostly incurable diseases like progressive neurological diseases, paraplegia and quadriplegia, HIV/ AIDS and severe strokes. Conservative estimates( as per WHO) 60 per cent of those dying are in need of some form of palliative care put the figure approximately 600,000 a year. With the rapid ageing of the population, the highest number of patients needing palliative care will, in the future, come from the senior citizens. The bright side of the scenario is that this country has a strong cultural and ritualistic community and family support system to tend its senior members. Family ties and family hierarchies remain strong in this country. Nevertheless, urbanization and mobility together with restrictions on family size and the expense of urban accommodation make for a loosening of these bonds, with increasing members of sick and elderly persons needing help and support from outside the family. A community oriented approach of palliative care with main stream health care integration can ultimately lead to a unique care model for its senior citizens. In order to solve the urgent issues of access and equity which always confront low and middle income countries and to address meaningful solutions of whole person care of a dying person and their family, we must move beyond the bed side. We must embrace the more penetrating models of community engagement in end of life care. The dying person cannot be viewed in isolation from their family, friends and community. Whilst symptom controls by doctors is essential, medical professionals can never address the myriad of social and existential problems alone that those facing death must contend with. We all need to acknowledge it fast that getting end of life care "right" lies at the heart of what it means to be a civilized society and this prioritizing this area need no apologies.

and Palliative Care Pharmacotherapy. Volume 17, Numbers 3/4 2003.

4. Non-communicable diseases in the South East Asia region, Situation and Response World Health Organization, 2011.

5. Smith Thomas j; Cassel Brian J-The economic challenges of Palliative Medicine. In Oxford Text Book of Palliative Medicine, Fourth Edition, Hanks G, Cherny N, Christaki N, Fallon M, Kaasa S, Porteoni K. Oxford University press, NY, 2010. 